\title{
Insight into the Compositional and Structural Nano Features of AIN/GaN DBRs by EELS-HAADF
}

\author{
Alberto Eljarrat, ${ }^{1, \star}$ Lluís López-Conesa, ${ }^{1}$ César Magén, ${ }^{2,3}$ Žarko Gačević, ${ }^{4}$ \\ Sergio Fernández-Garrido, ${ }^{4, \dagger}$ Enrique Calleja, ${ }^{4}$ Sónia Estradé, ${ }^{1,5}$ and Francesca Peiró ${ }^{1}$ \\ ${ }^{1}$ Laboratory of Electron NanoScopies, LENS-MIND-IN2UB, Dept. Electrónica, Universitat de Barcelona, Marti i Franqués 1, \\ 08028 Barcelona, Spain \\ ${ }^{2}$ Laboratorio de Microscopías Avanzadas (LMA), Instituto de Nanociencia de Aragón (INA), Departamento de Física de la \\ Materia Condensada, Universidad de Zaragoza, 50018 Zaragoza, Spain \\ ${ }^{3}$ Fundación ARAID, 50018 Zaragoza, Spain \\ ${ }^{4}$ Instituto de sistemas optoelectrónicos y Microtecnología (ISOM), Universidad Politécnica de Madrid, Ciudad Universitaria \\ s/n, 28040 Madrid, Spain \\ ${ }^{5}$ TEM-MAT, (CCiT), Universitat de Barcelona, Lluís Solé i Sabarís 1-3, 08028 Barcelona, Spain
}

\begin{abstract}
III-V nitride (AlGa)N distributed Bragg reflector devices are characterized by combined high-angle annular dark-field (HAADF) and electron energy loss spectroscopy (EELS) in the scanning transmission electron microscope. Besides the complete structural characterization of the AlN and GaN layers, the formation of AlGaN transient layers is revealed using Vegard law on profiles of the position of the bulk plasmon peak maximum. This result is confirmed by comparison of experimental and simulated HAADF intensities. In addition, we present an advantageous method for the characterization of nano-feature structures using low-loss EELS spectrum image (EEL-SI) analysis. Information from the materials in the sample is extracted from these EEL-SI at high spatial resolution.The log-ratio formula is used to calculate the relative thickness, related to the electron inelastic mean free path. Fitting of the bulk plasmon is performed using a damped plasmon model (DPM) equation. The maximum of this peak is related to the chemical composition variation using the previous Vegard law analysis. In addition, within the context of the DPM, information regarding the structural properties of the material can be obtained from the lifetime of the oscillation. Three anomalous segregation regions are characterized, revealing formation of metallic $\mathrm{Al}$ islands.
\end{abstract}

Key words: EELS, HAADF, III-V nitrides, DBR, STEM

\section{INTRODUCTION}

The analysis of electron energy loss spectroscopy (EELS) in the scanning transmission electron microscope (STEM) has turned out to be crucial for materials science. This is mainly because of the simultaneous reduction of the characteristic scale for device configuration and the technical improvements in microscopy that have occurred particularly during the past few years (Egerton, 2009). When EELS experiments are performed in the adequate conditions, each spectrum is related to the electronic, structural, and chemical properties of the examined material in a quantitative way. Moreover, when the electron probe being used is very spatially localized, so is the EELS information. In this work, computational tools are applied to analyze EELS spectrum images (EEL-SI), in which each pixel corresponds to a spatially localized spectrum, to extract the electronic and structural properties of the studied materials. Among these tools, nonlinear fitting of the plasmon peak using a damped oscillation model will allow the construction of profiles and maps of the plasmon peak width and position of the peak maximum. Calculations involving a linear relation between

Received November 23, 2012; accepted February 11, 2013

*Corresponding author. aeljarrat@el.ub.edu

${ }^{\dagger}$ Current address: Paul-Drude-Institut für Festkörperelektronik, Hausvogteiplatz 57, 10117 Berlin, Germany these positions and the composition of the layers will give chemical information about the examined materials. In addition, atomic model-based exit wave reconstruction simulations will confirm our calculations.

A major milestone in the development of blue-violet light emission by solid-state devices was achieved in the early 1990s, representing the breakthrough of group III nitrides in the materials science world as a new research hot topic (Nakamura et al., 1997; Ikeda \& Uchida, 2002). Thanks to their widely tunable room temperature band gap energy, these materials promise design of optoelectronic devices that cover the entire visible range. Nevertheless, in-plane lattice mismatch between the binary components is an issue affecting their growth, and defects and/or chemical segregations are usual, finally decreasing the overall performance of the devices (Wu et al., 2011). In this work, we deal with the structural and chemical characterization of a heterostructure of the binaries AlN/GaN for distributed Bragg reflectors (DBR). These are interesting materials for the construction of this device because of their relatively high refractive index contrast (Brunner et al., 1997), plus design and growth simplicity. The properties of the DBR originate from the distribution of staked layers of the binary materials, at the nanometer scale. To fully understand the properties of the examined samples, we need a technique capable of characterizing materials at this $\mathrm{nm}$ 
scale. Our strategy for this characterization is to simultaneously acquire low-loss EELS and Z-contrast high-angle annular dark-field (HAADF) images in an aberrationcorrected STEM.

\section{Materials And Methods}

\section{Molecular Beam Epitaxy (MBE) Growth}

For the configuration of the AlN/GaN DBR device, we consider a 20-period (AlGa) $\mathrm{N}$ multilayer structure designed to present peak reflectivity centered around $\sim 400 \mathrm{~nm}$. Semi-periods thicknesses were set to be $49.3 / 41.0 \mathrm{~nm}$ (AlN/ $\mathrm{GaN}$ ) to obtain the desired reflectivity. The growth was carried out in a RIBER Compact 21 MBE system equipped with a radio frequency plasma nitrogen source and standard Knudsen cells for gallium and aluminum (RIBER S.A., Bezons, France). Both GaN and AlN layers are grown below $\mathrm{GaN}$ decomposition threshold temperature, at $720^{\circ} \mathrm{C}$. To improve AlN crystalline quality, Ga should be used as surfactant. The examined structures have been grown following a carefully designed strategy in this sense, to reconcile the contradictory demands in MBE growth. A detailed description of the growth mechanism will be presented in an article in preparation.

\section{Sample Preparation and HAADF-STEM Observation}

The examined 20-period sample was prepared for STEMEELS observation by conventional mechanical polishing and $\mathrm{Ar}+$ ion milling, as described elsewhere (Gačević et al., 2010; Eljarrat et al., 2011, 2012). First, a preliminary examination at a JEOL J2010 F (S)TEM with a Schottky field emission gun (JEOL Ltd, Tokyo, Japan) operated at $200 \mathrm{keV}$ was performed.

A probe-corrected FEI Titan 60-300 (S) TEM operated at $300 \mathrm{kV}$ and equipped with a Wien filter monochromator (FEI Company, Hillsboro, OR, USA) and a Gatan Tridiem 866 ERS energy filter/spectrometer (Gatan Inc, Pleasanton, CA, USA) have been used to obtain the data from the sample at great spatial resolution. Features in the EEL spectrum, such as the plasmon excitation peak, can be measured at $\Delta E \simeq 0.2 \mathrm{eV}$ and $\Delta x \simeq 0.5 \mathrm{~nm}$ (nearly atomic column level) as indicated by the broadening of the zeroloss peak (ZLP), full-width at half-maximum and the most recent literature concerning the localization of the plasmon interaction (Dorneich et al., 1998; Brockt \& Lakner, 2000; Lazar et al., 2003; Arbiol et al., 2009; Benaissa et al., 2009).

EEL-SI datacubes were acquired, allowing us to calculate chemical, structural, and electronic properties in spatially localized maps for a whole identification of the layer stack and growth irregularities. Simultaneously acquired, the HAADF images were used to complement the EELS information.

\section{Characterization Through the Plasmon Peak}

The plasmon peak in the EEL spectrum is originated by the excitation of an electronic collective oscillation. In metals, it is modeled as an oscillation of the electrons in the conduction band, following the free electron model. In dielectrics and semiconductors (as in the present case), the plasmon can be modeled as a damped oscillation of the valence electrons, following the valence-resonance extension of the free electron model (Egerton, 2011).

As a common procedure when analyzing plasmon losses in ternary III-V nitride compounds, the first step has been to use the linear relation between the peak maximum and the binary composition through Vegard law. The precise position of the bulk plasmon peak was determined by Lorentzian fitting after Fourier-log deconvolution of the spectra, independently of the energy channel binning. All the specific computer routines for calculation have been implemented in Octave (Eljarrat et al., 2011, 2012). Fingerprinting was then performed by plasmon position variation profiling along the spectrum lines in combination with the simultaneously acquired HAADF intensity (see Fig. $2 \mathrm{~b}$ for a comparison between both profiles).

In order to extract more quantitative information from the analysis of the EEL-SI, we have followed a more theoretical approach to the problem using the damped plasmon model (DPM) described in equation (1) (Kundmann, 1988; Sánchez et al., 2005; Egerton, 2011). There are two parameters in the model function. One is related to the position of the peak, the modified plasma energy, $E_{\mathrm{P}}^{\prime}$, including the effects of band transitions and core electrons to the plasmon excitation. The other one is related to the broadening of the peak, $\Gamma$, also measured in $\mathrm{eV}$ :

$$
I(E) \propto \frac{E}{\left(E_{\mathrm{P}}^{\prime 2}-E^{2}\right)^{2}+E^{2} \Gamma^{2}} .
$$

Additional formulas for the calculation of parameters associated with the model can be found in the paper by Sánchez et al. (2005). We use them to calculate the lifetime for the plasmon pseudoparticle, $\tau=\hbar / \Gamma$, and the position of the peak maximum, $E_{\mathrm{MAX}}\left(E_{\mathrm{P}}^{\prime}, \Gamma\right)$ :

$$
E_{\mathrm{MAX}}\left(E_{\mathrm{P}}^{\prime}, \Gamma\right)=\left(\frac{\left(E_{\mathrm{P}}^{\prime}\right)-\Gamma^{2}+\sqrt{\left(2 E_{\mathrm{P}}^{\prime}-\Gamma^{2}\right)^{2}+12 E_{\mathrm{P}}^{\prime 4}}}{6}\right)^{1 / 2} .
$$

In parallel to this analysis, the log-ratio method has been applied to calculate thickness to inelastic mean free path ratios, $t / \lambda$. In this method, the elastic (the ZLP) and the inelastic (band transitions, plasmons, and/or core transitions) parts of the EELS spectrum are spliced and integrated. The logarithm of the ratio between these two integrals is equal to the relative thickness, $t / \lambda$, as explained in Egerton (2011).

Finally, EELS-based procedures have been complemented with atomistic model (Perez-Omil, 1994; Bernal et al., 1998)-based simulations of the HAADF intensities using exit wave reconstruction algorithms (Kirkland, 2010).

In summary, the experimental and computational methodology of this work can be described as follows: first, spectrum lines have been used to establish the relation 

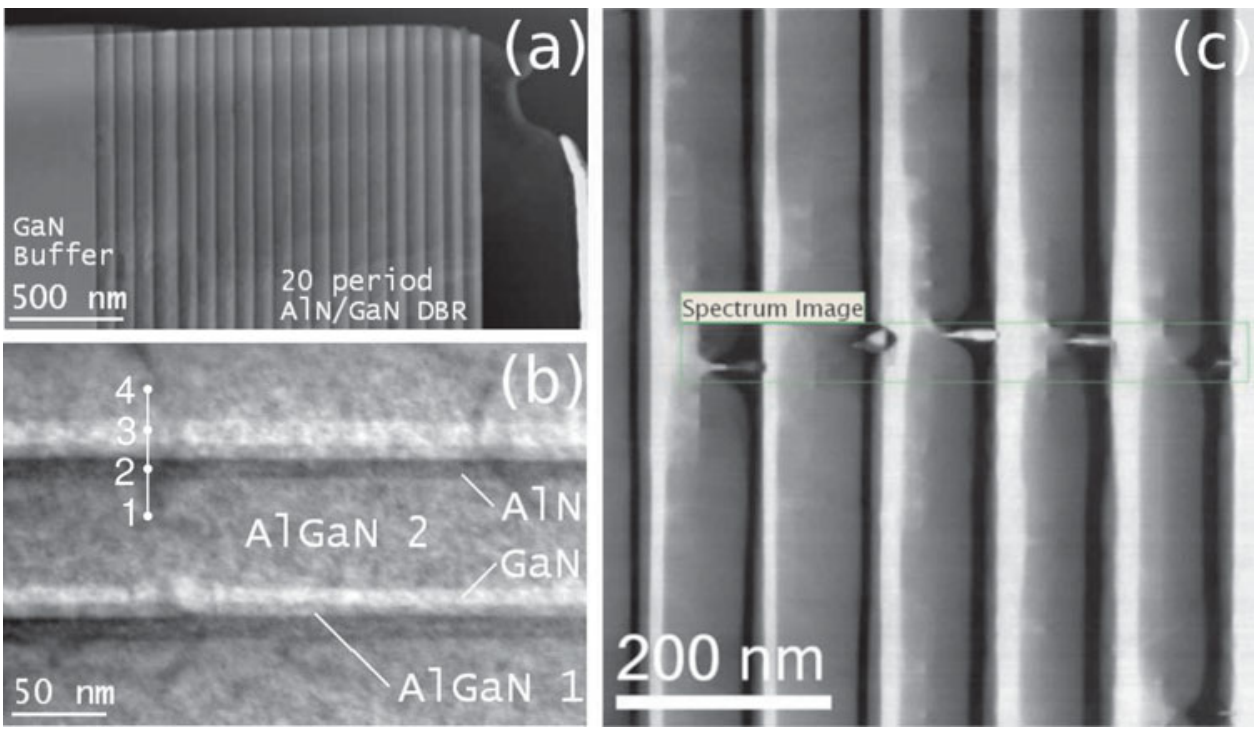

Figure 1. a: High-angle annular dark-field scanning transmission electron microscope of 20-period (AlGa) $\mathrm{N}$ full structure. The high periodicity of the structure is appreciated in this image, while (b) shows a detail of two successive periods. Four layers of different contrast are clearly visible, and their compositions based on Z contrast are annotated. The origin of the spectra from Figure 2 is indicated with numbered dots and a line. Finally, (c) presents the detail of an area were anomalous segregations are detected, and its surroundings. The green square indicates the region where the electron energy loss spectroscopy spectrum image of Figure 3 was acquired.

between the plasmon position of the peak maximum and the layer composition according to Vegard law between the binaries. Second, HAADF contrast simulations from atomistic models based in previous calculations of composition and thin foil thickness have been performed in order to compare them with the experimental HAADF intensity profiles and, hence, to validate the estimated composition derived from plasmon energy location. Finally, the DPM has been applied to a set of different EEL-SI obtained in regions exhibiting growth irregularities, and maps of composition and plasmon lifetime have been depicted for all these anomalous growth features.

\section{Results}

\section{Previous Characterization}

Optical and X-ray techniques were used for a macroscopic characterization of the samples. A noticeable deviation below the theoretically expected values was revealed in the reflectivity of the samples, 92 out of $99 \%$, measured by spectrophotometry. No cracks were found by optical microscopy examination of the structure. X-ray diffraction reciprocal space mapping measurements hinted the presence of AlGaN inclusions. An exhaustive account of these results will be presented in a parallel work.

Figure 1a displays the HAADF-STEM image of the full sample. A highly periodical structure, stable for several microns, is confirmed. At higher magnification, HAADF observations reveal that the (AlGa) N heterostructures appear to be composed of a periodic four-layer structure, allegedly AlGaN1 (AlN-on-GaN), GaN, AlGaN2 (GaN-onAlN), and AlN layers (see Fig. 1b). Yet, the chemical compo- sition of these layers, using only Z-contrast images, is not determined. Moreover, some anomalous segregations were detected in localized regions of the sample (see Fig. 1c).

\section{Determination of the Composition}

The continuous line through a whole period of the stack in Figure $1 \mathrm{~b}$ marks the line along which a typical EEL spectrum line was measured. In Figure 2a, four single spectra corresponding to the highlighted points $1-4$ are shown as an example. In Figure $2 b$, the position of the plasmon energy in comparison with the experimental HAADF intensity profile are shown for the whole spectrum line.

Given that the plasmon excitation energy will be modulated by the binary nitride composition of the $\mathrm{Al}_{x} \mathrm{Ga}_{(1-x)} \mathrm{N}$ layer and assuming a lineal relation between the plasmon excitation energy and the ratio of each component through a Vegard-like law of the form (Palisaitis et al., 2011):

$$
E_{\mathrm{P}}^{\mathrm{AlGaN}}=x E_{\mathrm{P}}^{\mathrm{AlN}}+(1-x) E_{\mathrm{P}}^{\mathrm{GaN}},
$$

with $E_{\mathrm{P}}^{\mathrm{A} 1 \mathrm{~N}}=21.1 \mathrm{eV}$ and $E_{\mathrm{P}}^{\mathrm{GaN}}=19.7 \mathrm{eV}$ being the bulk plasmon peak energies of the binary components (Dorneich et al., 1998; Brockt \& Lakner, 2000; Lazar et al., 2003; Arbiol et al., 2009; Benaissa et al., 2009), the aluminum nitride composition can be determined. Assuming the validity of equation (3) for the whole sample, and once the plasmon position profiles have been processed using Vegard law, the composition on one of these periods is well described by the variation of $x$, the aluminum nitride composition, along the spectrum line.

This result is depicted for a one-period profile (spectrum line in Fig. 1b), along with the experimental HAADF intensity, for comparison, in Figure 3a. With these profiles, 

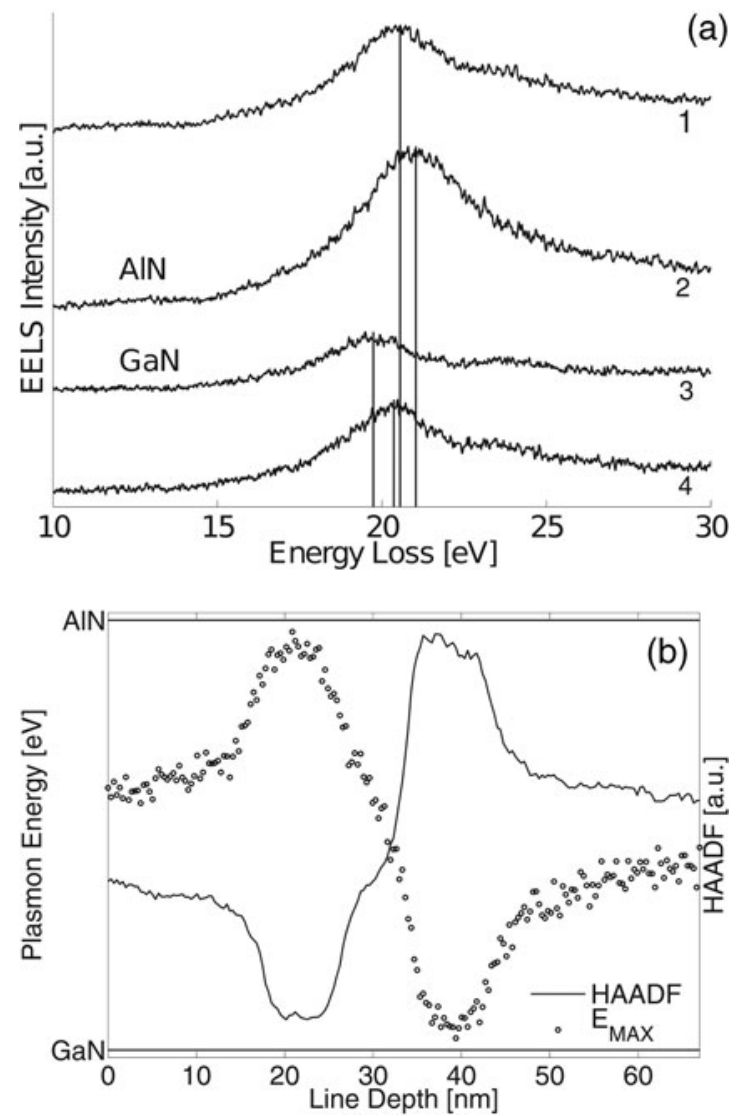

Figure 2. a: Four electron energy loss spectra from different regions of the layer, with a line showing their determined plasmon maxima and two annotations over the spectra taken from pure $\mathrm{AlN}$ and GaN regions are shown. In addition, the positions have been linked with Figure $1 \mathrm{~b}$ through numbers. b: Determined plasmon energy, $E_{\mathrm{MAX}}$ (circles), and the high-angle annular darkfield intensity (line) profiles through one period. These signals have been centered and normalized to allow comparison of the contrast that they provide.

it is possible to distinguish each of the layers that compose the repeating four-layer heterostructure. We are able to resolve the GaN, AlN, and intermediate $\mathrm{AlGaN}$ regions and to read from the profile the composition variation.

The experimental HAADF profile is shown in Figure $3 \mathrm{~b}$. Notice the gradual intensity variation due to thickness in this profile. Sample relative thickness, $t / \lambda$, were also calculated using the EEL spectra. The obtained profiles point to an increase of sample thickness along the direction of the line scan (as we approach the buffer layer), a quite appreciable $\sim 40 \%$ mean variation after three DBR periods, as expected from sample preparation and the modulation of the HAADF-integrated signal.

HAADF images were simulated using the compositions, thickness, and expected width of the layers obtained from EELS analysis as an input for a repeating four-layer structure. An atomic model for three periods, with the expected thickness variation, was created using the Rhodius software (copyright Universidad de Cádiz, Cádiz, Spain; Perez-Omil, 1994; Bernal et al., 1998). Then the STEM
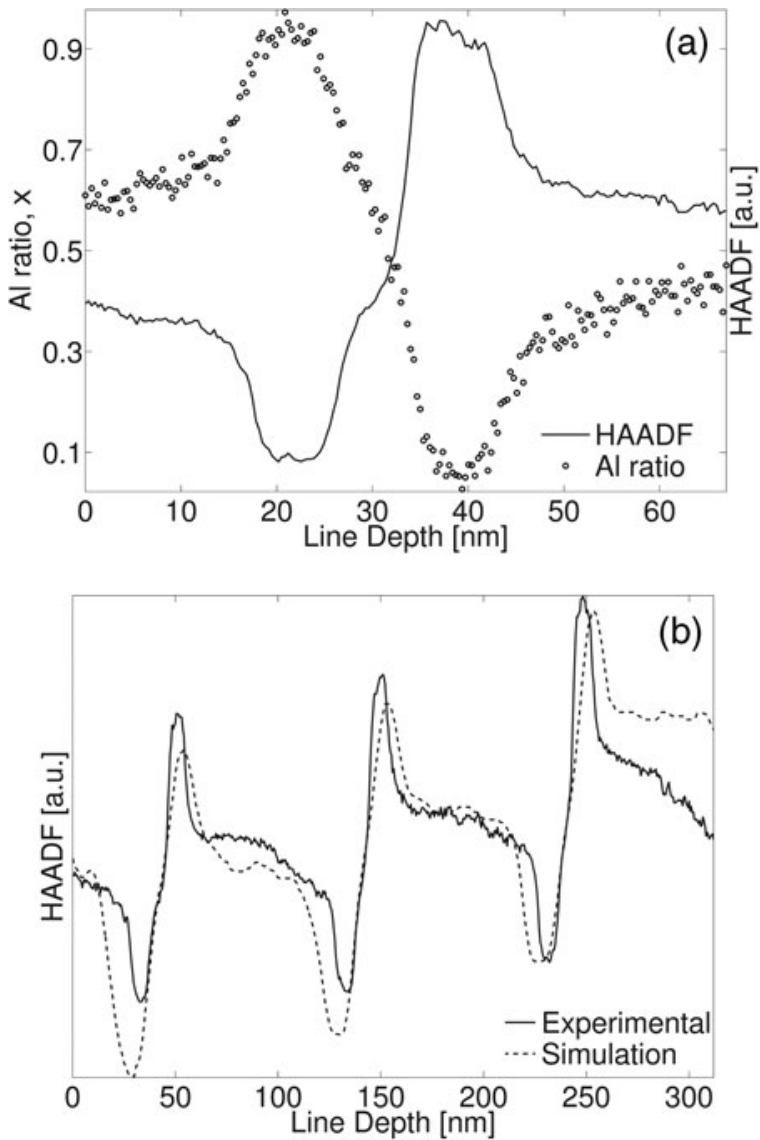

Figure 3. a: Aluminum ratio (circles) profiles calculated through Vegard law analysis of the plasmon excitation energy position and the high-angle annular dark-field (HAADF) intensity profile (line) corresponding to the spectrum line from 1 to 4 in Figure $1 \mathrm{~b}$ are shown. b: HAADF-integrated intensity profiles, experimentally acquired and simulated, along three periods are compared.

probe propagation through the atomic potential was calculated by the multislice method using the TEM-SIM software (available at the webpage of Cornell University, Ithaca, NY, USA, and Kirkland, 2010). The simulated integrated intensity profiles are also shown in Figure $3 \mathrm{~b}$ as a dotted line. As a linear variation in thickness was assumed in the simulations for simplicity, there is not a perfect agreement between simulated and experimental data. However, the simulation shows the expected thickness dependence of the HAADF signal and a fairly good accordance of the intensity variation due to composition.

\section{Mapping of Properties}

We now focus our attention on the anomalous segregations noticed in some regions of the heterostructure. HAADF images and EELS spectra in two dimensions (SI) were simultaneously acquired. The first region (see Fig. 4) appears as a nanometer-size bulging of the $\mathrm{GaN}$ layer, judging by the $\mathrm{Z}$ contrast in the HAADF image. In the case of the other two probed regions (see Figs. 5, 6 for the second one, and Fig. 7 for the third), segregations in greater extent were measured. We can observe, in these HAADF images, that 

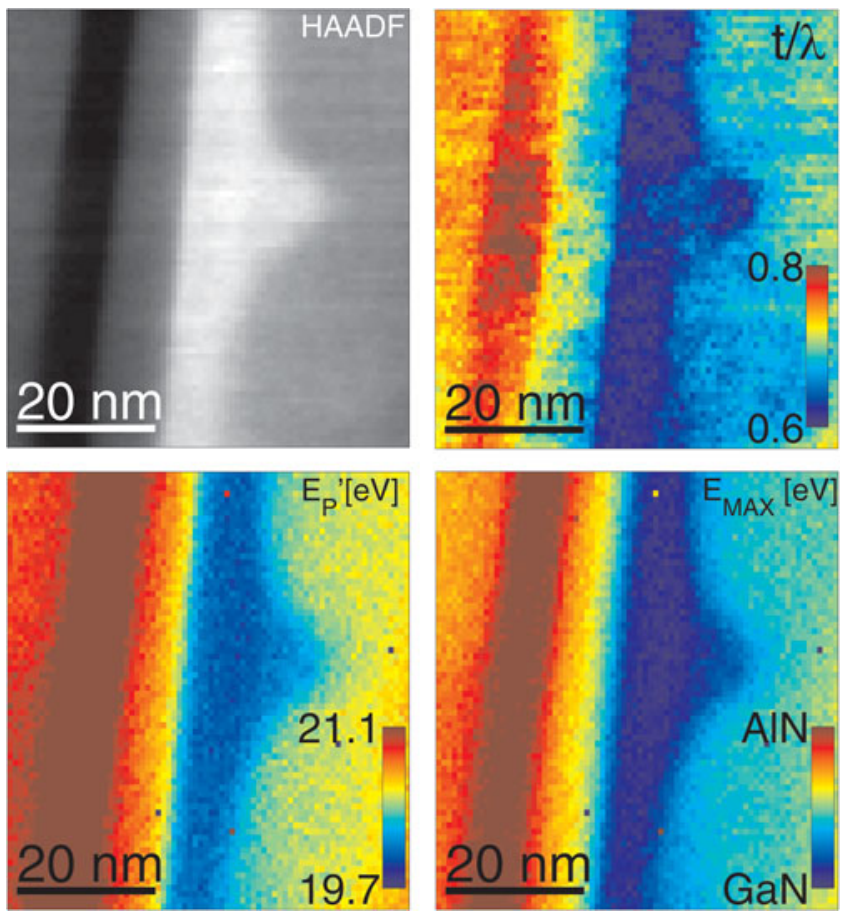

Figure 4. High-angle annular dark-field (HAADF)image and electron energy loss spectroscopy-calculated property maps for the first examined anomalous segregation region. Three maps are presented: the upper-right panel shows the relative thickness map and the two below show the damped plasmon model energy $E_{\mathrm{P}}^{\prime}$ and the plasmon maxima map. It can be appreciated that the pictures from the panels are quite similar.

the shape of the structure has been much altered, with segregations affecting consecutive layers. This collection of HAADF images (gray-scale panels) and maps of calculated properties (color-scale panels) depicting the three different examined regions will be presented in this section.

EEL-SI from these three different regions were analyzed using a nonlinear fitting to the DPM and the relative thickness, $t / \lambda$, was calculated. The DPM fit outputs two parameters, $E_{\mathrm{P}}^{\prime}$ and $\Gamma$. Using the associated formulas included in the model, we can recover the position of the peak maximum, $E_{\mathrm{MAX}}\left(E_{\mathrm{P}}^{\prime}, \Gamma\right)$, and the lifetimes of the plasmon pseudoparticle, $\tau(\Gamma)$. The Lorentzian formula applied to the spectrum lines in the last section fitted the peak's maximum energy position, $E_{\mathrm{MAX}}$. However, $E_{\mathrm{MAX}}$ was also retrieved from the two DPM parameters and the results showed a total correspondence with the Lorentzian fits in all cases.

The calculated maps $\left(t / \lambda, E_{\mathrm{P}}^{\prime}\right.$, and $E_{\mathrm{MAX}}$ parameters $)$ and the corresponding HAADF image from the first segregation region are presented in Figure 4. There is a striking resemblance between the images in all three panels in spite of the different procedures involved and the nature of the information displayed. The results from the fits also produce a map of $\Gamma$, related with the damping of the plasmon. In the case of this region, an almost homogeneous map of $\Gamma \simeq 5 \mathrm{eV}(\tau \simeq 0.13 \mathrm{fs})$ was produced, not shown here because it did not display any feature from the structure.
Observing the HAADF image of the second anomalous segregation region and the corresponding plasmon energy position map (see Fig. 5), we can appreciate how the GaN material of one period has been clamped down into the AlN layer of the next period. The simulation of the HAADF signal using an atomic model constructed with the compositions derived from the plasmon maps and Vegard law (also Fig. 5) confirms the correspondence between these two maps. The corresponding $t / \lambda$ and $\tau$ maps are presented in Figure 6: on one hand, the $t / \lambda$ of the material, through the inelastic electron mean free path, $\lambda$, shows two regions, higher/lower relative thickness or shorter/longer $\lambda$ (red/ blue in the image). On the other, it seems that not much information can be inferred from the blurry $\tau$ map, but on the central region there is a hint of a low plasmon-lifetime area (high damping, blue again). When compared with the other maps and the HAADF image, two parallel stripes are found, lying on the AlN/GaN interface.

Finally, Figure 7 shows a HAADF image of a segregation spanning for various periods, dramatically reshaping a region almost $500 \mathrm{~nm}$ long and $80 \mathrm{~nm}$ wide. The $t / \lambda$ map shows the red/blue region that seems to indicate two repeating constant $\lambda$ regions. In addition, this property is affected by the variation of thickness that occurs naturally because of the wedge shape of the sample, offsetting the contrast between the lateral ends. In the plasmon energy map, two island-shaped regions draw our attention to the center of the image. The plasmon energy inside these two islands is $\sim 15 \mathrm{eV}$, well below the rest of the map, where it lies between the pure GaN and AlN plasmon peak energies. This map, once again, shows a good correspondence with the HAADF signal, allowing for the chemical characterization of the region. In the lowermost panel, the $\tau$ map, the island regions are still visible but show a different type of contrast. The origin of these features in the map is a consistent broadening of the plasmon around the island and a narrowing inside. This is interpreted in the model as a decrease of $\tau$ around the island and an increase inside. This measured value for the plasmon energy plus the narrowing of the peak suggest metallic Aluminum composition of these islands.

\section{DISCUSSION}

From the HAADF images in Figure 1 we know that we have a highly stable four-layer periodic heterostructure. The composition can be directly read from Figure 3a (see also Fig. 1b, for visual reference). From right to left in Figure 3 (corresponding to the scanned line from 1 to 4 in Fig. 1) we can distinguish the transition corresponding to the ending of the AlGaN2 layer, with $x \simeq 0.4$, at a line depth of $\sim 15 \mathrm{~nm}$. Two nearly pure binary layers are detected after this, $x \simeq 0.1$ and $x \simeq 0.9$, with the narrow AlGaN1 layer between them, $x \simeq 0.5$. Finally, the beginning of the next AlGaN2 layer (this is a periodic structure) has a composition of $x \simeq 0.6$. In fact, the AlGaN2 layer appears to have a graded composition, with lower AlN content at the ends. 

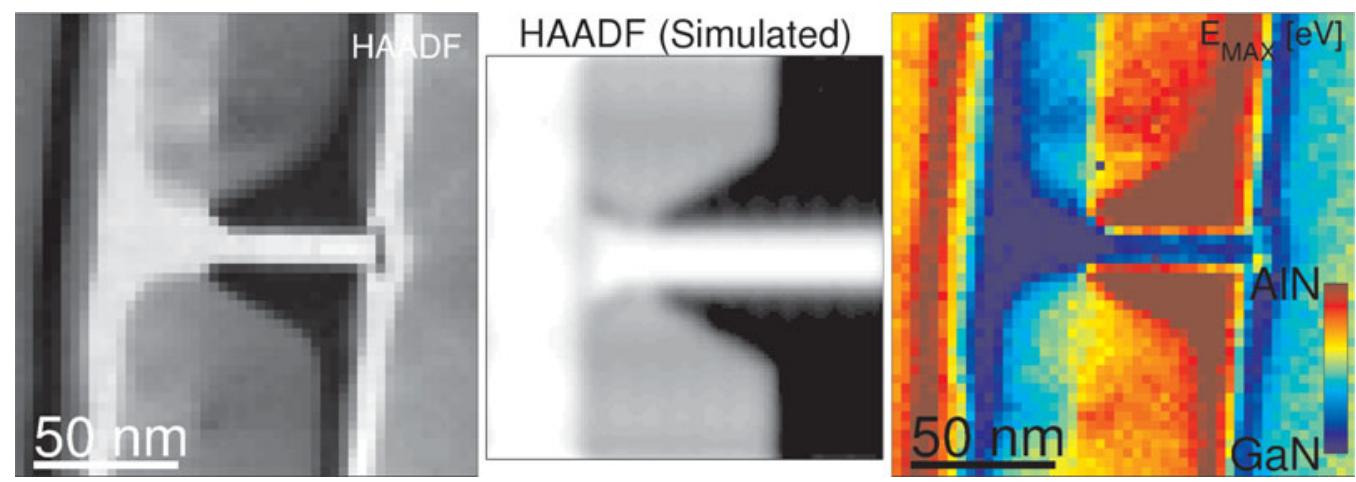

Figure 5. High-angle annular dark-field (HAADF) images, experimental and simulated, along with the plasmon peak maximum $\left(E_{\mathrm{MAX}}\right)$ map for the second examined segregation. The experimental HAADF and $E_{\mathrm{MAX}}$ show good correspondence again; the simulated HAADF is in good accordance too. This exit wave reconstruction simulation (size $363 \times 356$ pixel) was performed using an atomistic model based on the chemical information from $E_{\mathrm{MAX}}$ and Vegard law. The increased abruptness of the AlN/GaN interface in the clamped region can be assessed.
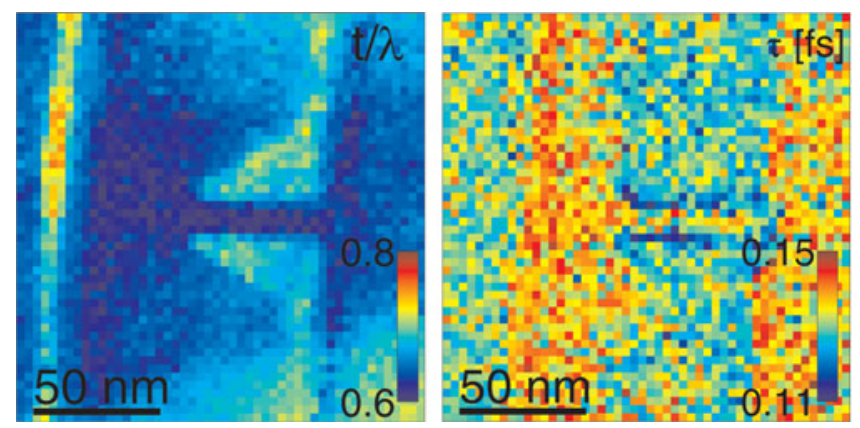

Figure 6. Two additional electron energy loss spectroscopycalculated property maps from the second examined segregation. The left panel shows the relative thickness map, which is quite distinct from the chemically sensitive pictures in Figure 5. The right one shows the damped plasmon model lifetime $\tau$ (with the parallel stripes-like region in the middle).
The average AlN content in the layers was calculated, finding $\langle x\rangle \simeq 0.5$ for all examined periods, in good accordance with nominal composition values from the growth. Finally, the width of the four layers that compose the periodic heterostructure is determined from EELS analysis and confirmed through simulation and comparison with the HAADF intensities: $\sim 10,15,50$, and $15 \mathrm{~nm}$ for AlGaN1 (AlN-on-GaN), GaN, AlGaN2 (GaN-on-AlN), and AlN layers, respectively.

Localized anomalous segregation regions were also observed in the DBR, and they have been studied through EEL-SI detailed analysis. In the case of the first anomalous region (see Fig. 4), there was a striking resemblance between the HAADF image, the $t / \lambda$, the $E_{\mathrm{P}}^{\prime}$, and the $E_{\mathrm{MAX}}$ maps. Note that, although the contrast for the modified electron plasma energy map, $E_{\mathrm{P}}^{\prime}$, is not exactly the same as the one
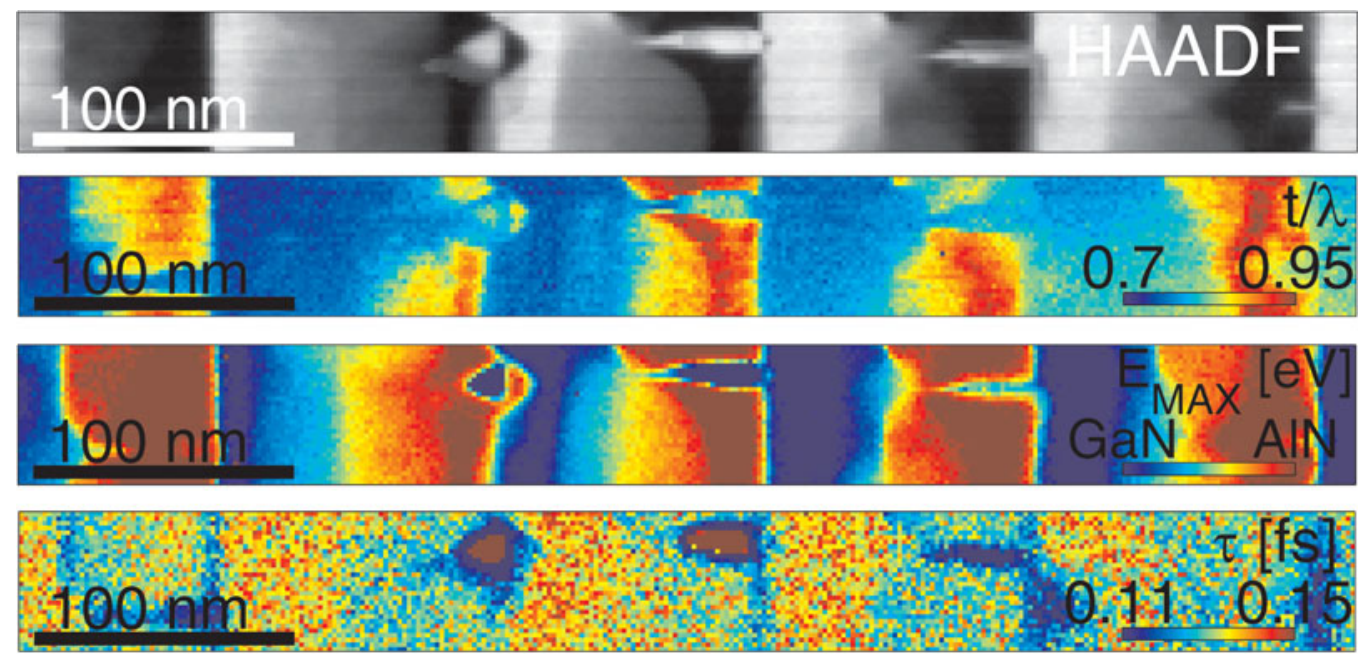

Figure 7. High-angle annular dark-field (HAADF) image and three electron energy loss spectroscopy-calculated property maps from the third EELS spectrum image, with the relative thickness, the $E_{\mathrm{MAX}}$ and the $\tau$. Notice the thickness contrast in the first map (second from the top), because of the increment in thickness from left to right of the sample (this is a long map). In addition, the metallic $\mathrm{Al}$ islands are noticeable in the $\tau$ image (halo-like region) and in the $E_{\mathrm{MAX}}$ (strong plasmon contrast); metallic $\mathrm{Al}$ has around $\sim 5 \mathrm{eV}$ plasmon energy difference compared with GaN or AlN. 
for the peak maximum, $E_{\mathrm{MAX}}$, this is expected in the context of the DPM. The plasmon energetic parameters, $E_{\mathrm{P}}^{\prime}$ and $E_{\mathrm{MAX}}$, are obtained through the same fitting procedure, and are related to the local chemical compositions. This fit procedure also produced the $\tau(\Gamma)$ parameter, but in this case the broadening of the plasmon could not be connected with any particular property of the examined region, as the maps were homogeneous.

The $t / \lambda$ parameter (relative thickness) should show contrast at nearly constant thickness because of the different electron mean free paths, $\lambda$, present in the region. This parameter is computed by a procedure that involves the ratio between integrated spliced parts of the EEL spectrum, as explained in the Characterization Through the Plasmon Peak section, which is remarkably different from the nonlinear fit procedure. We can conclude that, for this region, the composition dependence of $\lambda$ is linear, as it is for the plasmon excitation energy or the HAADF intensity.

This similar behavior of the HAADF intensity and the plasmon peak maximum energy is assessed again in the second anomalous segregation region (see Fig. 5), with a more complex structure and features with reduced size. An atomic model was built using the information in the plasmon position map and, through exit wave reconstruction simulation, the contrast in the HAADF image was simulated. Note that, because of computational limitations, only a fraction of the structure that is seen in the images has been simulated. The simulation recreates the HAADF contrast from a GaN layer being clamped into the next period AlN layer, with good correspondence with the experimental HAADF image.

Two additional maps were presented for this region (see Fig. 6), with different information in them. The map for the $t / \lambda$ parameter in this region now shows a contrast, which deviates from the linear behavior that was observed in the previous region. Note that the $t / \lambda \mathrm{pa}-$ rameter is in the same range as it was in the previous region (see Fig. 4), indicating that the absolute thickness was similar. But this time, although the contrast for the AlN layers is preserved, the $\mathrm{GaN}$ and $\mathrm{AlGaN}$ regions appear blurred out in the map. The $\tau$ maps for this region show a contrast, which is related to the damping of the plasmon oscillation. In this case, the abruptness of the AlN/GaN interface is greatly increased, with an effect on the measured $\Gamma$ and $\tau(\Gamma)$ parameters from the interface region. The maximum of $\Gamma$ and minimum of plasmon lifetime, $\tau$, appear to lie close to the interface. This is just where the maximum strain gradient in the material should be placed, as the lattice mismatch between both AlN and GaN materials is great.

The third of our examined segregations spanned through five periods (see Fig. 7). The thickness increase from left to right due to the wedge shape of the prepared sample can be correlated with the gradual variation of the $t / \lambda$ map. Moreover, the good correspondence between HAADF features and the $t / \lambda$ map also points toward a linear depen- dence on composition for almost the whole map, except in the islands regions. Larger nearly pure $\mathrm{AlN}$ and $\mathrm{GaN}$ layers were found in this region, and, according to the plasmon map obtained for this region, we found what appeared to be two metallic islands of pure Al. These islands cannot be easily identified in the $t / \lambda$ map, but are clearly characterized by the plasmon energy of pure $\mathrm{Al}, \sim 15 \mathrm{eV}$. What is even more surprising is the fact that the $\tau$ parameter is low around the island and higher inside it, confirming the existence of a halo-like signal surrounding the island. The possibility that a halo-like area for the $\Gamma$ parameter surrounded strained or amorphized areas was mentioned by other authors (Kundmann, 1988; Sánchez et al., 2005).

In brief, plasmon damping, measured through the $\Gamma$ parameter of the plasmon fit, showed halo-like contrast only in situations where the structure had unusually abrupt interfaces, AlN-to-GaN or AlN-to-Al. Although we can rely on the compositional sensitivity of the plasmon peak, our analysis indicates that the $\tau$ parameters may have a great structural sensitivity. Moreover, for the latter two segregations, linear contrast correspondence is lost in the $t / \lambda$ maps. The plasmon energy and HAADF contrast are strongly correlated with the composition, although, as other authors have already pointed out (Iakoubovskii \& Mitsuishi, 2008), an extensive study of the $\lambda(Z)$ behavior is needed in order to predict the dependence of this parameter.

\section{CONCLUSIONS}

In summary, in this work, we have proposed a useful method for characterizing materials using the combination of STEM-HAADF and low-loss EEL-SI mapping and we have applied it to characterize a III-V nitride (AlGa) N DBR device. The obtained EELS data have been analyzed through various techniques. We have presented phenomenological (such as Vegard law) and theoretical (such as the DPM) formulations, used to retrieve information and gain insight into the sample properties. In addition, we have compared our data with exit wave reconstruction simulations of HAADF images as a sanity check. The calculations have been performed using homemade routines for automatic EEL-SI data analysis. In this way, it was possible to retrieve the plasmon energy position, and thus the local chemical composition. Also, the plasmon damping has allowed us to obtain physical information contained in the plasmon peak, but not reflected in the position of the maximum. Finally, relative thickness has been calculated, which we have shown to reveal thin foil sample variations and linear/nonlinear dependence of $t / \lambda$ on composition.

The combination of EELS and HAADF in the STEM has proved to be a valuable tool in the characterization of structural properties from local measurements at great spatial resolution and chemical sensibility. The interpretation of the maps constructed from the calculated parameters opens new possibilities for the application of EELS to device analysis. 


\section{ACKNOWLEDGMENTS}

Electron microscopy characterization has been carried out using the TEM facilities of the "Scientific and Technological Centres" (University of Barcelona) and the "Laboratorio de Microscopias Avanzadas" at "Instituto de Nanociencia de Aragon" (Universidad de Zaragoza). The authors acknowledge the LMA-INA for offering access to their instruments and expertise. This work was carried out within the framework of MAT-2010-16407 and CSD-2009-00013 projects from Spanish Ministerio de Ciencia e Innovación, also CAM (P2009/ESP-1503) project and CTP2011-00018 and 2009SGR00035 from Catalan Government.

\section{ReFERENCES}

Arbiol, J., Estrade, S., Prades, J.D., Cirera, A., Furtmayr, F., Stark, C., Laufer, A., Stutzmann, M., Eickhoff, M., Gass, M.H., Bleloch, A.L., Peiro, F. \& Morante, J.R. (2009). Tripletwin domains in $\mathrm{Mg}$ doped GaN wurtzite nanowires: Structural and electronic properties of this zinc-blende-like stacking. Nanotechnology 20(14), 145704-145713.

Benaissa, M., Gu, L., Korytov, M., Huault, T., Van Aken, P.A., Brault, J. \& Vennegues, P. (2009). Phase separation in GaN/ AlGaN quantum dots. Appl Phys Lett 95(14), 141901-141904.

Bernal, S., Botana, F., Calvino, J., Lopez-Cartes, C., PerezOmil, J. \& Rodriguez-Izquierdo, J. (1998). The interpretation of HREM images of supported metal catalysts using image simulation: Profile view images. Ultramicroscopy 72(3-4), 135-164.

Brockt, G. \& Lakner, H. (2000). Nanoscale EELS analysis of dielectric function and bandgap properties in $\mathrm{GaN}$ and related materials. Micron 31(3), 435-440.

Brunner, D., Angerer, H., Bustarret, E., Freudenberg, F., Hopler, R., Dimitrov, R., Ambacher, O. \& Stutzmann, M. (1997). Optical constants of epitaxial AlGaN films and their temperature dependence. J Appl Phys 82(10), 5090-5097.

Dorneich, A.D., French, R.H., Müllejans, H., Loughlin, S. \& RüHLE, M. (1998). Quantitative analysis of valence electron energy-loss spectra of aluminium nitride. J Microsc 191, 286-296.

Egerton, R.F. (2009). Electron energy-loss spectroscopy in the TEM. Rep Prog Phys 72, 016502-016527.

Egerton, R.F. (2011). Electron Energy-Loss Spectroscopy in the Electron Microscope. New York: Springer.

Eljarrat, A., Gačević, Ž., Fernández-Garrido, S., Calleja, E., Magén, C., Estradé, S. \& Peiró, F. (2011). (V)EELS character- ization of InAlN/GaN distributed Bragg reflectors. J Phys Condens Matter 326(1), 012014-012018.

Eljarrat, A., Gačević, Ž., Fernández-Garrido, S., Calleja, E., Magén, C., Estradé, S. \& Peiró, F. (2012). Optoelectronic properties of InAlN/GaN distributed Bragg reflector heterostructure examined by valence electron energy loss spectroscopy. Microsc Microanal 18(5), 1143-1154.

GaČević, Ž., Fernández-Garrido, S., Hosseini, D., Estradé, S., Peiró, F. \& Calleja, E. (2010). InAlN/GaN Bragg reflectors grown by plasma-assisted molecular beam epitaxy. J Appl Phys 108, 113117-113124.

Iakoubovsкiı, K. \& Mitsuishi, K. (2008). Mean free path of inelastic electron scattering in elemental solids and oxides using transmission electron microscopy: Atomic number dependent oscillatory behavior. Phys Rev B 77 (10), 104102-104109.

IKedA, M. \& UCHIDA, S. (2002). Blue violet laser diodes suitable for Blu-ray disk. Phys Status Solidi 194, 407-413.

Kirkland, E.J. (2010). Advanced Computing in Electron Microscopy. New York: Springer.

Kundmann, M. (1988). Study of semiconductor valence plasmon line shapes via electron energy-loss spectroscopy in the transmission electron microscope. PhD Thesis. Berkeley, CA: University of California at Berkeley.

Lazar, S., Bотtom, G.A., Wu, M.-Y., TichelaAr, F.D. \& ZandberGEN, H.W. (2003). Materials science applications of HREELS in near edge structure analysis and low-energy loss spectroscopy. Ultramicroscopy 96(3-4), 535-546.

Nakamura, S., Pearton, S. \& Fasol, G. (1997). The Blue Laser Diode. The Complete Story. New York: Springer.

Palisaitis, J., Hsiao, C.-L., Junai, M., Xie, M., Darakchieva, V., Carlin, J.-F., Birch, N.G.J., Hultman, L. \& Persson, P.O.A. (2011). Standard-free composition measurements of $\mathrm{Al}_{x} \mathrm{In}_{1-x} \mathrm{~N}$ by low-loss electron energy loss spectroscopy. Phys Stat Sol RRL 5(2), 50-52.

Perez-Omil, J. (1994). Interpretación sistemática de imágenes de microscopía electrónica de alta resolución de materiales policristalinos. Estudio de catalizadores metálicos soportados. PhD Thesis. Cádiz, Spain: University of Cadiz.

Sánchez, A.M., Beanland, R., Gass, M.H., Papworth, A.J., Goodhew, P.J. \& Hopkinson, M. (2005). Mapping quantum dot-in-well structures on the nanoscale using the plasmon peak in electron energy loss spectra. Phys Rev B 72(7), 075339-075347.

Wu, C.M., Zhang, B.P., Shang, J.Z., Cai, L.E., Zhang, J.Y., Yu, J.Z. \& WANG, Q.M. (2011). High-reflectivity AlN/GaN distributed Bragg reflectors grown on sapphire substrates by MOCVD. Semicond Sci Tec 26, 055013-055018. 